\title{
Mutagenic activity and metabolites in the urine of workers exposed to trinitrotoluene (TNT)
}

\author{
G AHLBORG JR, ${ }^{2}$ PIRKKO EINISTÖ, ${ }^{2}$ MARJA SORSA ${ }^{2}$
}

From the Department of Occupational Medicine, '̈ Örebro Medical Centre Hospital, Regionsjukhuset, S-701 85 Örebro, Sweden, and Department of Industrial Hygiene and Toxicology, Institute of Occupational Health, SF-00290 Helsinki 29, Finland

\begin{abstract}
Urine samples taken after work and after a free weekend from 50 workers employed in various activities in a chemical plant manufacturing explosives were analysed. On the basis of hygienic surveys, the subjects were divided into three categories of exposure to trinitrotoluene (TNT). The urine analyses consisted of gas chromatographic identification of TNT and its two metabolites, 4-ADNT and 2-ADNT, and a determination of the mutagenic activity. Two frame shift detector strains of Salmonella typhimurium were used, TA 98 and TA 98 NR, the latter being deficient in endogenous nitroreductase activity. On the basis of previous results on TNT mutagenicity, no exogeneous metabolic system was used to test the urine concentrates. Both tester strains showed that the mean urinary mutagenic activity was higher in the after work samples than in post weekend samples from the same subjects, showing that bacterial nitroreductase activity was not significantly responsible for the mutagenicity, although the response was higher with strain TA 98 than with TA 98 NR. The interindividual variation in urine mutagenicity was high, however, and the difference between the two sampling times was statistically significant $(p<0.05)$ only for the high exposed group (workers in trotyl foundry and sieve house). Correlation between urinary mutagenicity and concentration of TNT in urine was poor; correlation was significant only with the urinary concentration of 4-ADNT. The correlation between urinary TNT and both metabolites was good $(p<0.001)$. These results suggest that analysis of 4-ADNT in urine would be a sufficient biological measure for controlling exposure to TNT.
\end{abstract}

In an earlier study we found that workers at a chemical plant producing explosives had increased mutagenic activity in the urine after exposure to trinitrotoluene (TNT). ${ }^{1}$ Mutagenic activity was determined in the bacterial fluctuation assay ${ }^{2}$ and the highest activity was obtained when the indicator strain Salmonella typhimurium TA 98 was used without exogeneous metabolic system added. This stain has some endogenous nitroreductase activity and thus the ability to reduce nitrocompounds to perhaps more mutagenic metabolites. Many nitroaromatic substances are mutagenic only to bacterial strains with this capability. ${ }^{3}$

Even though TNT has been used as a military explosive since the beginning of this century, few studies have been published of its metabolism in man. ${ }^{45}$ Quantitatively, 4-aminodinitrotoluene (4ADNT) seems to be the dominating metabolite, but unmetabolised TNT can be found in the urine of

Accepted 11 May 1987 exposed workers (P Einistö et al, International symposium on biochemical and cellular indices of human toxicity in occupational and environmental medicine, Milan, 1986). The main route of biotransformation is believed to be nitroreduction but oxidation is also possible. The metabolites are excreted in the urine in the free and conjugated forms.

The toxic effects of TNT were noted during the first world war when many TNT workers were reported as dying from aplastic anaemia or toxic hepatitis. ${ }^{6}$ Other toxic effects of TNT are gastritis, dermatitis, cataract, and haemolytic anaemia. Human data concerning eventual genotoxic effects are not available, but in a pilot study we found an unexpectedly high incidence of stomach cancer among TNT workers.?

Since TNT is easily absorbed through the skin biological monitoring of workers would be a preferable means of assessing exposure compared with environmental monitoring. The aim of the present study was to assess the mutagenic activity in the urine of workers at different levels of exposure and to 
analyse TNT and its metabolites 4-ADNT and 2-ADNT in the same samples to find out a possible correlation between these parameters. Furthermore, the aim was to evaluate which analysis would be best suited for biological assessment of exposure.

\section{Study group and sampling}

All production workers at the plant working in foundries or assembly departments where TNT was handled were asked to give two urine samples; one after a free weekend (unexposed sample) and the other at the end of a workshift (exposed sample). Personnel at the controlling and experiment departments and laboratory workers in contact with TNT were also asked to participate. Only a few refused. A few others were able to deliver only one urine sample before they were assigned to another job. A total of 50 (19 women and 31 men) delivered both an unexposed and an exposed sample (table 1).

On both days of sampling, each worker completed a questionnaire concerning smoking habits and alcohol consumption, diet, health, and medication. Special attention was paid to the 48 hours preceding sampling.

The "unexposed samples" were collected in the morning after a free weekend before the worker entered the work area. The "exposed samples" were collected immediately after a workshift. All samples were collected in plastic bottles and divided into several portions before freezing to $-20^{\circ} \mathrm{C}$, at which temperature they were transported to the laboratory and kept until analysed.

Blood samples were taken on the same day as the exposed samples and analysed for liver enzymes (S-GOT, S-GPT, S-ALP, and S-GT).

\section{Exposure}

TNT is produced at the plant and used in grenades and

Table 1 Number of workers giving urine samples and measured TNT concentration for each department

\begin{tabular}{|c|c|c|c|c|}
\hline Group & Department & Total No & Exposed* & $\begin{array}{l}T N T / \\
\text { concentration } \\
\left(\mathrm{mg} / \mathrm{m}^{3}\right) \dagger\end{array}$ \\
\hline $\begin{array}{l}\text { A } \\
\text { B } \\
\text { C } \\
\text { D } \\
\text { E } \\
\text { F } \\
\text { G }\end{array}$ & $\begin{array}{l}\text { Trotyl foundry } \\
\text { Sieve house } \\
\text { Test foundry } \\
\text { Octol-hexotol foundry } \ddagger \\
\text { Assembling of grenades } 1 \\
\text { Laboratories } \\
\text { Controlling and } \\
\text { experiment }\end{array}$ & $\begin{array}{l}5 \\
5 \\
2 \\
8 \\
15 \\
9 \\
6\end{array}$ & $\begin{array}{r}5 \\
5 \\
2 \\
6 \\
12 \\
4 \\
3\end{array}$ & $\begin{array}{l}0,2-0,5 \\
0,5 \\
0,2-0,3 \\
0,1-0,2 \\
0,1 \\
-\end{array}$ \\
\hline
\end{tabular}

*TNT work was performed during the shift preceding the exposed sample.

†Personal sampling in the breathing zone, mainly performed in June 1983.

$\ddagger$ Mixtures of explosives including TNT. mines, in some cases mixed with other explosives such as hexaminetetranitrate. The explosives are formed into different sizes and shapes in the foundries to fit the various shells.

Measurements of air concentrations of TNT have been performed several times at the worksites. Some additional measurements were made in June 1983 and the results of these are shown in table 1. In the laboratories and the controlling and experiment departments only small quantities of TNT were handled and the air concentrations were considered too low for the analytical detection limit. Casting and cooling were the processes emitting the highest concentrations of TNT vapour whereas the highest concentrations of TNT dust were found in the sieve house. The workers used protective clothing and had access to respirators when performing work operations involving risks of heavy exposure.

On the basis of previous hygienic surveys, the subjects were divided into three categories of TNT exposure:

1 No or low exposure (groups $F$ and $G$ )

2. Medium exposure (groups $C, D$, and E)

3 High exposure (groups A and B).

During the workshift preceding the exposed sample all deviations from normal production were recorded such as interruptions or leakages. Twelve individuals were actually found not to have been in contact with TNT during the shift, four of whom were not working in the laboratories or in the controlling and experiment departments; despite this they were included in exposure category 1 .

\section{Methods and exclusions}

\section{BACTERIAL MUTAGENICITY ASSAY OF URINE} SAMPLES

To decrease interindividual variability in counting the mutagenic activities, the urine samples were adjusted to a creatinine concentration of $4 \mathrm{mmol} / \mathrm{l}$ before they were concentrated on XAD-2 resin. The bacterial fluctuation test was then performed in the same way as in our previous study.' Two frame shift indicator strains were used, Salmonella typhimurium TA 98 and its homologue Salmonella typhimurium TA 98 NR, the latter being deficient in nitroreductase activity. On the basis of previous results, no exogeneous metabolic system was added. All samples were analysed blindly on codes.

The mutagenic activity was calculated as follows:

Mutagenic activity $=\frac{\left(\operatorname{Rev}_{\mathrm{s}}-\operatorname{Rev}_{\mathrm{c}}\right) \mathrm{k}}{\text { Creatinine conc }}$

$\operatorname{Rev}_{\mathrm{s}}=$ revertants in sample; $\operatorname{Rev}_{\mathrm{c}}=$ revertants in solvent control; $\mathbf{k}=$ dilution factor. Creatinine concentration $=4 \mathrm{mmol} / \mathrm{l}$. 
ANALYSES OF TNT, 4-ADNT, AND 2-ADNT

2,4,6-TNT, 4-ADNT, and 2-ADNT were analysed in the same urine samples using the method described by Allmog et al. ${ }^{8}$ A portion of the urine samples were concentrated on XAD- 2 resin in the same way as in the mutagenicity assay. One millilitre of concentrate was adjusted to $\mathrm{pH} 2$ with diluted HCL. After ten minutes the sample was neutralised with a surplus of sodium bicarbonate followed by extraction with $1 \mathrm{ml}$ toluene. The water phase was eliminated and water free sodium sulphate was added to the toluene phase. One microlitre of the clear toluene extract was injected into a Hewlett-Packard 5790 gas chromatograph with ${ }^{63} \mathrm{Ni}$-EC-detector. A fused silica capillary column $12 \mathrm{~m}$, OV 101 was used. Injection temperature $200^{\circ} \mathrm{C}$, detection temperature $330^{\circ} \mathrm{C}$, and temperature programme $60^{\circ}-200^{\circ}\left(5^{\circ}\right.$ a minute). Helium was used as carrier gas. Calibration with external standard (2,4,6-TNT, 4ADNT, and 2-ADNT). The results are presented in micromoles per mol creatinine; concentrations below detection limit have been given a value of zero in the calculations.

\section{EXCLUSIONS}

The same restrictions as in our previous study of urine mutagenicity were used.' Urine samples with an original creatinine concentration lower than 3.0 $\mathrm{mmol} / \mathrm{l}$ were excluded to avoid overestimation of the mutagenic activity. Individuals with the highest values in each of the liver enzymes analysed (S-GOT, S-GPT, S-ALP, or S-GT) were also excluded. Due to these restrictions five individuals were not included in the results (two because of diluted urine and three because of abnormally high liver enzymes).

In two cases the urine samples were toxic to the indicator bacteria even at the lowest concentration tested. Thus 43 individuals remained for calculating the results.

\section{STATISTICAL METHODS}

For each exposure group the mean $(\overline{\mathbf{x}})$, the median (Md), and the range of mutagenic activity, TNT, 4-ADNT, and 2-ADNT was calculated. Since the number of observations in each category was relatively small and the underlying distributions deviated from the normal, non-parametric tests of significance were applied. ${ }^{9}$ Wilcoxon's signed rank test was used to test the difference between exposed and unexposed samples. When testing the difference between the exposure categories Wilcoxon's rank sum test was used. The $5 \%$ level has been applied with two tailed calculations $(p<0.05)$. The coefficient of correlation (r) was calculated as an expression of the relation between the analysed factors.

\section{Results}

\section{MUTAGENIC ACTIVITY}

The mutagenic activity was higher in the exposed than in the unexposed samples; in TA 98 mean 340 (Md 40, range $0-3040)$ and 73 (Md 20, range 0-600) respectively; in TA $98 \mathrm{NR}$ mean 162 (Md 100, range 0-800) and 60 (Md 20, range 0-440).

Table 2 gives the results for each exposure group. Highly increased urine mutagenicity in the exposed samples was found only among workers in the trotyl foundry and in the sieve house, most pronounced when tested with TA $98(\mathrm{p}<0.05)$. The difference between the exposed and unexposed samples in exposure categories 1 and 2 was insignificant.

Table 2 Mutagenic activity (St TA 98 and TA 98 NR; revertants/mmol creatinine) in urine from TNT workers after a free weekend (unexposed) and after a workshift (exposed). Mean $(\bar{x})$, median (Md), and range

\begin{tabular}{|c|c|c|c|c|c|c|c|}
\hline \multirow[b]{2}{*}{ Group } & \multirow[b]{2}{*}{ No } & \multicolumn{3}{|c|}{ Unexposed sample } & \multicolumn{3}{|c|}{ Exposed sample } \\
\hline & & $\bar{x}$ & $M d$ & Range & $\bar{x}$ & $M d$ & Range \\
\hline $\begin{array}{l}\text { TA } 98 \\
\text { A } \\
\text { B } \\
\text { C } \\
\text { D } \\
\text { E } \\
\text { F } \\
\text { G } \\
\text { Unexposed* }\end{array}$ & $\begin{array}{r}5 \\
5 \\
2 \\
4 \\
10 \\
4 \\
3 \\
10\end{array}$ & $\begin{array}{r}208 \\
116 \\
0 \\
15 \\
66 \\
25 \\
167 \\
22\end{array}$ & $\begin{array}{r}40 \\
20 \\
0 \\
0 \\
50 \\
0 \\
20 \\
0\end{array}$ & $\begin{array}{l}0-600 \\
0-440 \\
0 \\
0-60 \\
0-180 \\
0-100 \\
0-480 \\
0-120\end{array}$ & $\begin{array}{r}1068 \\
1424 \\
160 \\
35 \\
118 \\
45 \\
33 \\
26\end{array}$ & $\begin{array}{r}1040 \\
880 \\
160 \\
20 \\
30 \\
10 \\
20 \\
30\end{array}$ & $\begin{array}{c}80-2720 \\
0-3040 \\
140-180 \\
0-100 \\
0-440 \\
0-160 \\
0-80 \\
0-60\end{array}$ \\
\hline $\begin{array}{l}\text { TA } 98 \text { NR } \\
\text { A } \\
\text { B } \\
\text { C } \\
\text { D } \\
\text { E } \\
\text { F } \\
\text { G } \\
\text { Unexposed* }\end{array}$ & $\begin{array}{r}5 \\
5 \\
2 \\
4 \\
10 \\
4 \\
3 \\
10\end{array}$ & $\begin{array}{r}104 \\
60 \\
150 \\
75 \\
34 \\
75 \\
0 \\
54\end{array}$ & $\begin{array}{r}20 \\
0 \\
150 \\
90 \\
30 \\
60 \\
0 \\
40\end{array}$ & $\begin{array}{c}20-440 \\
0-220 \\
140-160 \\
0-120 \\
0-80 \\
0-180 \\
0 \\
0-140\end{array}$ & $\begin{array}{r}124 \\
480 \\
120 \\
120 \\
148 \\
245 \\
33 \\
66\end{array}$ & $\begin{array}{r}140 \\
560 \\
120 \\
110 \\
140 \\
90 \\
0 \\
0\end{array}$ & $\begin{array}{r}0-260 \\
120-800 \\
80-160 \\
100-160 \\
0-300 \\
0-800 \\
0-100 \\
0-400\end{array}$ \\
\hline
\end{tabular}

*TNT work was not performed during the workshift preceding the exposed sample. 
Table 3 Mutagenic activity (St TA 98 and TA 98 NR; revertants/mmol creatinine), TNT, 4-ADNT, and 2-ADNT ( $\mu$ mol/mol creatinine) in urine from smokers $(n=22)$ and non-smokers $(n=21)$. Mean $(\bar{x})$, median $(M d)$, and range

\begin{tabular}{|c|c|c|c|c|c|c|}
\hline \multirow[b]{2}{*}{ Analyses } & \multicolumn{3}{|c|}{ Smokers } & \multicolumn{3}{|c|}{ Non-smokers } \\
\hline & $\bar{x}$ & Md & Range & $\bar{x}$ & $M d$ & Range \\
\hline $\begin{array}{l}\text { Unexposed sample } \\
\text { TA } 98 \\
\text { TA } 98 \mathrm{NR}\end{array}$ & $\begin{array}{l}86 \\
65\end{array}$ & $\begin{array}{l}50 \\
30\end{array}$ & $\begin{array}{l}0-480 \\
0-440\end{array}$ & $\begin{array}{l}60 \\
56\end{array}$ & $\begin{array}{r}0 \\
20\end{array}$ & $\begin{array}{l}0-600 \\
0-220\end{array}$ \\
\hline $\begin{array}{l}\text { Exposed sample } \\
\text { TA 98 } \\
\text { TA 98 NR } \\
\text { TNT } \\
\text { 4-ADNT } \\
\text { 2-ADNT }\end{array}$ & $\begin{array}{l}417 \\
181 \\
0.07 \\
13.35 \\
3.11\end{array}$ & $\begin{array}{c}50 \\
140 \\
0.07 \\
3.92 \\
1.23\end{array}$ & $\begin{array}{l}0-2800 \\
0-800 \\
0-0 \cdot 31^{*} \\
0 \cdot 58-72 \cdot 24^{*} \\
0-24 \cdot 43^{*}\end{array}$ & $\begin{array}{l}260 \\
142 \\
0.22 \\
37.67 \\
7.88\end{array}$ & $\begin{array}{c}40 \\
100 \\
0.07 \\
9.07 \\
1.70\end{array}$ & $\begin{array}{l}0-3040 \\
0-720 \\
0-2 \cdot 44 \\
0-218 \cdot 14 \\
0-65 \cdot 28\end{array}$ \\
\hline
\end{tabular}

None of the differences between smokers and non-smokers is statistically significant ( $p>0.05)$.

${ }^{*} \mathrm{n}=20$.

Smokers did not have significantly higher mutagenic activity in urine than non-smokers (table 3). An exogeneous metabolic system needs to be added to the test system for the mutagenic effect of smoking to appear clearly.'

TNT, 4-ADNT, AND 2-ADNT

To get an idea of possible background "noise," unexposed samples from seven individuals in different exposure categories were analysed for TNT, 4-ADNT, and 2-ADNT. In none of these was any of the substances detectable. The volumes of two exposed

Table $4 T N T, 4-A D N T$, and 2-ADNT ( $\mu \mathrm{mol} / \mathrm{mol}$ creatinine) in urine from TNT workers after a work shift (exposed sample). Mean $(\bar{x})$, median $(M d)$, and range

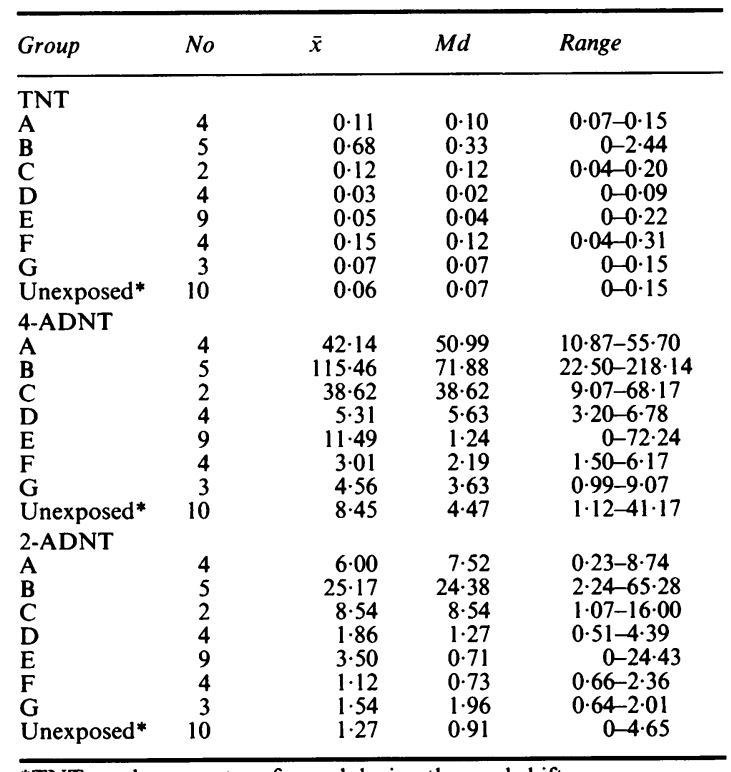

*TNT-work was not performed during the workshift. samples were too small to yield enough concentrate for the analyses and therefore the results include 41 individuals. The mean concentration of all the samples was $0 \cdot 15 \mu \mathrm{mol} \mathrm{TNT} / \mathrm{mol}$ creatinine (Md 0.07, range $0-2.44), \quad 25.80 \mu \mathrm{mol} \quad 4-\mathrm{ADNT} / \mathrm{mol}$ creatinine (Md 5.84, range $0-218.14$ ) and $5.55 \mu \mathrm{mol}$ $2-\mathrm{ADNT} / \mathrm{mol}$ creatinine (Md 1.24, range 0-65.28). 4-ADNT was quantitatively the dominant metabolite.

The sieve house group had the highest concentrations on average of all three substances in the exposed urine samples. Also the trotyl foundry and test foundry group showed higher mean values than the other groups (table 4). The difference between exposure categories 3 and 1 is statistically significant: TNT mean 0.42 and $0.08 \mu \mathrm{mol} / \mathrm{mol}$ creatinine (p < 0.05); 4-ADNT mean 82.87 and $6.49 \mu \mathrm{mol} / \mathrm{mol}$ creatinine ( $<<0.001) ; 2$-ADNT mean 16.65 and 1.28 $\mu \mathrm{mol} / \mathrm{mol}$ creatinine $(\mathrm{p}<0.001)$.

Non-smokers had somewhat higher values in the exposed samples than smokers but this difference was not statistically significant $(\mathrm{p}>0.05)$.

\section{CORRELATION BETWEEN MUTAGENICITY AND METABOLITES}

The correlation between the mutagenic activity in the urine and its content of TNT, 4-ADNT, and 2-ADNT was generally relatively weak and statistically significant only for 4-ADNT ( $p<0.05$, table 5). This

Table 5 Relation (coefficient of correlation) between mutagenic activity (TA 98 and TA $98 \mathrm{NR}$ ), TNT, 4-ADNT, and 2-ADNT in urine from 41 workers after workshift (exposed sample)

\begin{tabular}{|c|c|c|c|c|}
\hline & TA 98 & $T A 98 N R$ & $T N T$ & 4-ADNT \\
\hline $\begin{array}{l}\text { TA } 98 \text { NR } \\
\text { TNT } \\
\text { 4-ADNT } \\
\text { 2-ADNT }\end{array}$ & $\begin{array}{l}0.60^{* * *} \\
0.08 \\
0.33^{*} \\
0.30\end{array}$ & $\begin{array}{l}0.11 \\
0.36^{*} \\
0.23\end{array}$ & $\begin{array}{l}0.73^{* * *} \\
0.90^{* * *}\end{array}$ & $0.90^{* * *}$ \\
\hline
\end{tabular}


was also true within exposure category 3 . The correlation between the metabolites were strong, however $(\mathrm{r}>0.70 ; \mathrm{p}<0.001)$. In exposure category 1 there was no significant correlation between TNT and the two metabolites but in the two other exposure categories there was a strong relation.

\section{Discussion}

The results from the tests of urine mutagenicity are in agreement with those in our previous study - namely, that the workers considered most highly exposed showed the highest mutagenic activity in the urine after a workshift. As expected, the Salmonella strain lacking nitroreductase activity (TA 98 NR) was less sensitive to these aromatic nitroaminocompounds. Nevertheless, even with this indicator bacterium the mutagenicity was higher in the exposed than in the unexposed samples, thus showing that bacterial nitroreductase activity is not significantly responsible for the mutagenicity arising from TNT exposure.

On the group level the agreement between mutagenicity and the amount of TNT metabolites found was fairly good. Only a small fraction of TNT which has entered the body is excreted unchanged. Of the two metabolites analysed in this study, 4-ADNT dominated quantitatively. Another perhaps equally important metabolite is 6-ADNT. ${ }^{10}$ The interindividual variation even within each exposure category was relatively large both with regard to mutagenic activity and the metabolites. In this study only a relative quantification of metabolites has been possible and the reason for this is discussed further below.

\section{MUTAGENIC ACTIVITY}

Determination of mutagenic activity in the bacterial fluctuation test is semiquantitative. Falck has estimated the limit value for a significantly increased mutagenic activity in the urine from several hundred non-exposed, non-smoking individuals to be about $600 \mathrm{rev} / \mathrm{mmol}$ creatinine with Salmonella typhimurium TA 98." Only the category with the highest TNT exposure (3) had a mean value over this level. Both the level and the variation of mutagenic activity in the unexposed samples were of the same magnitude as in our previous study.' The interindividual variation within each exposure group is due not only to biological differences such as toxicokinetics or biotransformation but also to differences in uptake since different individuals within the same workplace differ with regard to work tasks or personal hygiene.

TNT, but not its metabolites including ADNT, was earlier shown to be mutagenic in the Ames' assay with Salmonella typhimurium TA $98 .^{12}$ Our finding of no correlation between mutagenic activity and TNT concentration (but a weak correlation with 4-ADNT) is therefore not easily explained apart from the fact that the TNT concentrations generally were very low and therefore variations negligible. It is possible that other mutagenic metabolites emanating from, for example, oxidative metabolic systems may contribute to urine mutagenicity among individuals with high exposure. Concomitant exposure to other substances such as hexamintetranitrate occurred in a few of the production departments. In our earlier study, however, the workers producing hexamintetranitrate did not show any increase in urine mutagenicity after exposure.

Concentration of potentially mutagenic compounds using the XAD-2 resin is by far the most frequently used system in urine mutagenicity studies. ${ }^{13}$ Recent recovery studies using radiolabelled (C 14) TNT in urine from rats have shown that only $20-60 \%$ appeared in the concentrate (P Einisto et al, Milan, 1986). The recovery was dependent on the original concentration and $\mathrm{pH}$ of the urine. Using the newly developed Bond Elut C-18 the recovery was $80-90 \%$ and the ability to remove histidine equal to XAD-2. Thus it seems as if XAD-2 would be less suited for the analyses of the compounds of interest in this study. Our results must therefore be regarded rather as an underestimation of the true mutagenic activity (and TNT concentration) in the exposed samples. On the group level, however, the results should be valid to indicate the relative magnitude of the TNT uptake.

\section{TNT, 4-ADNT, AND 2-ADNT}

In the analyses of some samples it was not possible to determine the concentration of TNT, 4-ADNT, or 2-ADNT because the levels did not significantly exceed the practical detection limit of the GC method. The lowest value received at a single analysis was 0.009 nmol TNT/ml concentrate. We did not detect any TNT, 4-ADNT, or 2-ADNT in the seven unexposed urine samples analysed. A few of the individuals who were thought not to have been exposed to TNT during the workshift after which the exposed samples were collected had detectable amounts of TNT and ADNT in urine. This may be explained by indirect exposure from work areas previously contaminated with TNT. There is also a possibility of individual toxicokinetic variation, which means that some individuals excrete TNT-metabolites for a longer period after exposure. ${ }^{10}$ It is not clear to what extent there are quantitative differences between individuals with regard to different metabolic pathways. More research is needed to clarify this.

In a recent study Wollen et al showed that the excretion of 4-ADNT and 6-ADNT in urine from exposed workers varied from one individual to the other. ${ }^{10}$ Excretion was highest at the end of the workshift but a few individuals had as high (or higher) 
concentration in the morning sample the day after exposure. In some cases ADNT could still be detected 17 days after exposure. There was no correlation between urine and air concentrations of TNT. When the uptake through inhalation was calculated based on the air concentrations a much lower figure was obtained than the total uptake estimated from the urine concentrations of ADNT. The authors therefore concluded that dermal absorption must contribute significantly to total uptake. The exposure categories used in our study not only differed with regard to air concentrations of TNT but also in the possibilities for contamination of the skin. Dermal uptake is most likely to occur among the workers exposed to high dust concentrations as, for example, in the sievehouse. The highest mutagenic activity and the highest metabolite concentrations were found in this group of workers.

In conclusion, it seems possible to use ADNT in urine (perhaps only 4-ADNT) as a biological indicator of exposure to TNT. It is, however, too early to suggest any biological limit value for the urinary metabolites of TNT; this was also the opinion of Wollen et al..$^{10}$ Since the dermal uptake probably is more important than the uptake through inhalation, a biological limit value would be highly desirable and perhaps possible to establish in the future. At present we recommend the use of ADNT in urine only at the group level to assess significant exposure and to investigate the effects of preventive hygienic measures. After the extensive improvement programme to minimise exposure to TNT has been carried through at the plant where this study was conducted, such an evaluation will be undertaken in the departments with previous high exposures.

This study was made possible by the wholehearted cooperation of the Safety Committee and the health personnel at Bofors Nobel Kemi. We thank Professor Christer Hogstedt from the National Board of Occupational Safety and Health, Solna, for good advice in the design of the study, and our colleagues,
Ms Beatrice Bäck, Ms Pirkko Pfäffli, and Ms Pirjo Taropainen from the Institute of Occupational Health, Helsinki, for help in carrying out the chemical analyses.

The study was supported by a grant from the Swedish Work Environment Fund.

\section{References}

1 Ahlborg G jr, Bergström B, Hogstedt C, Einistö P, Sorsa M. Urinary screening for potentially genotoxic exposures in a chemical industry. Br J Ind Med 1985;42:691-9.

2 Green MHL, Muriel WJ, Bridges BA. Use of a simplified fluctuation test to detect low levels of mutagens. Mutat Res 1976;38:33-42.

3 Rosenkranz EJ, McCoy EC, Mermelstein R, Rosenkranz HS. Evidence for the existence of distinct nitroreductases in salmonella typhimurium-roles in mutagenesis. Carcinogen 1982; 5:121-3.

4 Channon BJ, Mills GT, Williams RT. The metabolism of 2,4,6trinitrotoluene (alpha-TNT). Biochem J 1944;38:70-85.

5 Lemberg R, Callaghan JP. Metabolism of aromatic nitrocompounds. 3. Isolation of reduction products of 2,4,6-trinitrotoluene from urine of rats and from human urine. Aust $J$ Exp Biol Med Sci 1945;23:13-20.

6 Hathaway JA. Trinitrotoluene: A review of reported dose-related effects providing documentation for a work place standard. $J$ Occup Med 1977;19:341-5.

7 Ahlborg G, Bryngelsson IL, Svensson B. Cancerincidens bland trinitrotoluen(TNT)-exponerade arbetare. Hygiea 1985; 94: 118.

8 Allmog J, Kraus S, Basch A. Determination of TNT-metabolites in urine. Arch Toxicol 1983;6:suppl:351-3.

9 Colton T. Statistics in medicine. Boston: Little Brown and Company, 1974.

10 Wollen BH, Hall MG, Craig R, Steel GT. Trinitrotoluene: assessment of occupational absorption during manufacture of explosives. Br J Ind Med 1986;43:465-73.

11 Falck K. Application of the bacterial urinary mutagenicity assay in detection of exposure to genotoxic chemicals. Helsinki: Institute of Occupational Health, 1982. (Academic dissertation.)

12 Won WD, DiSalvo LH, $\mathrm{Ng} \mathrm{J}$. Toxicity and mutagenicity of $2,4,6$ trinitrotoluene and its microbial metabolites. Appl Environ Microbiol 1976;31:576-80.

13 Vainio H, Sorsa M, Falck K. Bacterial urinary assay in monitoring exposure to mutagens and carcinogens. In: Berlin A. Draper M, Hemminiki K, Vainio H., eds. Monitoring human exposure to carcinogenic and mutagenic agents, IARC sci publ 1984; 59:247-58. 